\title{
Anti-doping analysis
}

\author{
Mario Thevis
}

Published online: 28 May 2011

(C) Springer-Verlag 2011

Sports drug testing has become a complex discipline facing numerous challenges, for example, the detection of prohibited drugs, the identification of prohibited methods, the discovery of manipulation, the assessment of analyte stability, etc. Because of the great variety of potential doping practices, doping-control laboratories utilize a broad spectrum of (bio) analytical approaches including (among others) mass spectrometry, isoelectric focusing/gel electrophoresis, and immunological methods. In this special issue, which is dedicated to modern anti-doping analysis, current state-of-the-art methodologies enabling comprehensive screening, sensitive confirmation procedures, metabolism studies, and quality assurance issues relevant to doping control are presented, and recent approaches and future perspectives are outlined.

Chromatography interfaced with mass spectrometry has been one of the most important tools in sports drug testing for decades, and the composition of this special issue of Analytical and Bioanalytical Chemistry further substantiates the fact that, in particular, liquid chromatography-(tandem) mass spectrometry (LC-MS(-MS)) with high-resolution, highaccuracy mass analyzers has gained considerable attention and use. It has been employed in comprehensive screening methods for low- and high-molecular-mass compounds and in metabolism studies of peptidic drugs, cannabimimetic compounds, stimulants, and selective estrogen receptor

Published in the special issue Anti-Doping Analysis with Guest Editor Mario Thevis.

M. Thevis $(\bowtie)$

German Sport University Cologne, Center for Preventive

Doping Research / Institute of Biochemistry,

Am Sportpark Muengersdorf 6,

50933 Cologne, Germany

e-mail: thevis@dshs-koeln.de modulators (SERMs). Moreover, its use to identify polysaccharide-based therapeutics has been demonstrated, and the identification of compounds indicative of autologous blood transfusion in urine specimens (i.e., urinary metabolites of phthalate-derived plasticizers) has been shown.

Complementary to mass spectrometric approaches, alternative strategies have been evaluated, tested, and implemented in routine doping control. Among these, detection methods for erythropoiesis-stimulating agents (ESAs, e.g., erythropoietin and its mimetics) are of particular importance and commonly rely on isoelectric focusing or SDSPAGE and immunoblotting. In addition, the misuse of human growth hormone (hGH) and its detection has been a major issue for sports drug-testing authorities, and, besides the determination of hGH isoform ratios that reveal the application of recombinant hGH, a so-called marker approach has been developed that focuses on two principal products stimulated by administration of hGH. Finally, a method referred to as surface plasmon resonance (SPR) has been demonstrated to have the potential to support antidoping efforts concerning several different analytes and could complement future drug-testing programs.

Besides sophisticated analytical chemistry, doping controls require stringent quality control and pre-analytical conditions. These are the subject of two contributions on the stabilization of urine specimens for doping control and the relevance of certified reference material to unambiguous identification and correct quantification of prohibited compound(s).

Overall, the breadth of anti-doping science requires multi-disciplinary approaches and the consideration of numerous aspects that ensure the highest-quality analysis. Hence, continuous effort to improve analytical standards and to expand test methods is necessary, and selected facets of research dedicated to sports drug testing are highlighted in this special issue. 


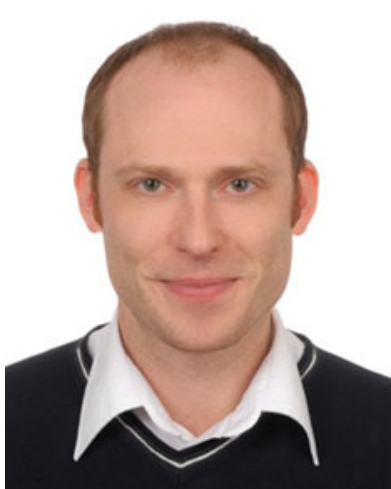

Mario Thevis is Professor at the German Sport University Cologne and Head of the Center for Preventive Doping Research since 2006. His research interests include the development of methods targeting non-approved emerging drugs with potential for misuse in sport, elucidation of metabolic pathways of drug candidates, and, particularly, the identification of peptidic drugs and their metabolites in blood and urine. He has published over 160 articles in peer-reviewed international journals, authored a book on mass spectrometry in sports drug testing, received the Manfred-Donike (2001) and Beynon-Price (2010) awards, and is Editor-in-Chief of Drug Testing and Analysis. 